International Journal of Modern Physics D

(C) World Scientific Publishing Company

\title{
ELLIPTICITY ANALYSIS OF THE BOOMERanG CMB MAPS
}

\author{
V. G. GURZADYAN,$^{*, \dagger}$ P. A. R. ADE ${ }^{\ddagger}$ P. De BERNARDIS,$\S$ C. L. BIANCO,${ }^{\dagger}$ \\ J. J. BOCK,$\ddagger$ A. BOSCALERI, $\|$ B. P. CRILL, ${ }^{* *}$ G. De TROIA,$\S$ K. GANGA, ${ }^{\dagger}$ \\ M. GIACOMETTI,$^{\S}$ E. HIVON,$^{\dagger \dagger}$ V. V. HRISTOV, ${ }^{* *}$ A. L. KASHIN, ${ }^{*}$ \\ A. E. LANGE,${ }^{* *}$ S. MASI,${ }^{\S}$ P. D. MAUSKOPF,${ }^{\ddagger}$ T. MONTROY,$\ddagger \ddagger$

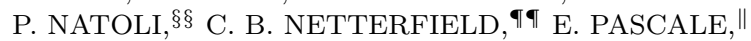 \\ F. PIACENTINI, ${ }^{\S}$ G. POLENTA ${ }^{\S}$ and J. RUHL ${ }^{\ddagger \ddagger}$ \\ * Yerevan Physics Institute, Armenia \\ †ICRA, Dipartimento di Fisica, Universita' La Sapienza, Roma, Italy \\ ${ }^{\ddagger}$ Department of Physics and Astronomy, Cardiff, UK \\ $\S$ Dipartimento di Fisica, Universita' La Sapienza, Roma, Italy \\ ฯ JPL, Pasadena, USA \\ "IROE-CNR, Firenze, Italy \\ ** Caltech, Pasadena, USA \\ ${ }^{\dagger} I P A C$, Pasadena, USA

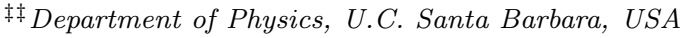 \\ $\S \S$ Dipartimento di Fisica, Tor Vergata, Roma, Italy \\ and \\ ฯ Department of Physics, University of Toronto, Canada
}

\begin{abstract}
The properties of the Cosmic Microwave Background (CMB) maps carry valuable cosmological information. Here we report the results of the analysis hot and cold CMB anisotropy spots in the BOOMERanG $150 \mathrm{GHz}$ map in terms of number, area, ellipticity, vs. temperature threshold. We carried out this analysis for the map obtained by summing independent measurement channels (signal plus noise map) and for a comparison map (noise only map) obtained by differencing the same channels. The anisotropy areas (spots) have been identified for both maps for various temperature thresholds and a catalog of the spots has been produced. The orientation (obliquity) of the spots is random for both maps. We computed the mean elongation of spots obtained from the maps at a given temperature threshold using a simple estimator. We found that for the sum map there is a region of temperature thresholds where the average elongation is not dependent on the threshold. Its value is $\sim 2.3$ for cold areas and $\sim 2.2$ for hot areas. This is a non-trivial result. The bias of the estimator is $\lesssim+0.4$ for areas of size $\lesssim 30^{\prime}$, and smaller for larger areas. The presence of noise also biases the ellipticity by $\lesssim+0.3$. These biases have not been subtracted in the results quoted above. The threshold independent and random obliquity behaviour in the sum map is stable against pointing reconstruction accuracy and noise level of the data, thus confirming that these are actual properties of the dataset. The data used here give a hint of high ellipticity for the largest spots. Analogous elongation properties of CMB anisotropies had been detected for COBE-DMR 4 year data. If this is due to geodesics mixing, it would point to a non zero curvature of the Universe.
\end{abstract}

Keywords: Cosmic microwave background. 


\section{Introduction}

The properties of the Cosmic Microwave Background (CMB) radiation continue to be a key window to our understanding of the early evolution and the present structure of the Universe. Recent experiments provided a detection of acoustic peaks in the angular power spectrum of the CMB, which is an important characteristic of the conditions at the last scattering epoch, and hence constrains a number of cosmological parameters (see e.g. Refs. 12, 13, 23, 24, 28, 29, and 34). Below we report the results of the analysis of the ellipticity of the anisotropies in the sky maps from BOOMERanG. At a given temperature threshold, spots with temperature higher (lower) than the threshold are identified. There are several definitions of the ellipticity of one spot, depending on the detailed procedure used to measure it. Loosely speaking, ellipticity is the ratio between the major and the minor semiaxes of the ellipse best fitting the contour of the spot. Ellipticity is expected in the CMB maps, as a result of the physical effects occurring before recombination, which induce correlations in the image of the CMB. This paper, however, is in the spirit of a model independent analysis of the data, so we will not attempt to compare to and constrain different models of the CMB anisotropy. Various descriptions have been proposed and already used for the study of CMB maps. The main aim was to check the Gaussian nature of the data (see e.g. Refs. [5, 8, 30, 33 and 39). Detailed numerical simulations of geometrical descriptions have been carried out in the framework of the Planck mission in Ref. 6] Particularly, the analysis of the shapes of anisotropies in the COBE-DMR maps has shown a signature of elongation, 21 which was absent in the maps containing only noise. The present analysis aimed to check the same effect in the BOOMERanG data. Since these data feature significantly higher angular resolution and lower noise than the COBE-DMR ones, 937 this goal is of particular interest.

The elongation analysis, which implies the estimation of Lyapunov exponents, was initiated with the aim to check the effect of geodesic mixing 18 which can cause additional elongation of CMB anisotropies independent on the temperature threshold. For the geodesics in hyperbolic spaces (Anosov systems) the scattering effectively occurs at every point of the space and in any two-dimensional directions. The resulting ellipticity has to be independent on the angular size of the spots. This is completely different from the result of gravitational lensing, the scattering on clumps of matter, which has been shown to produce a distortion of CMB spots only at sub-degree scales. The elongation of anisotropies due to the contribution of the noise, on the other hand, has to be threshold dependent $[$ The estimation of Lyapunov exponents, Kolmogorov-Sinai (KS) entropy and similar geometrical characteristics has proved its efficiency in the analysis and interpretation of various experimental results in a variety of fields (see e.g. Refs. 15, 35 and 36). In the following we show that the BOOMERanG sum map (A + B hereafter, see Fig. 1) reveals a threshold independent elongation of the anisotropies, which are more homogeneous with respect to the difference map (A - B hereafter, see Fig. 2). The 

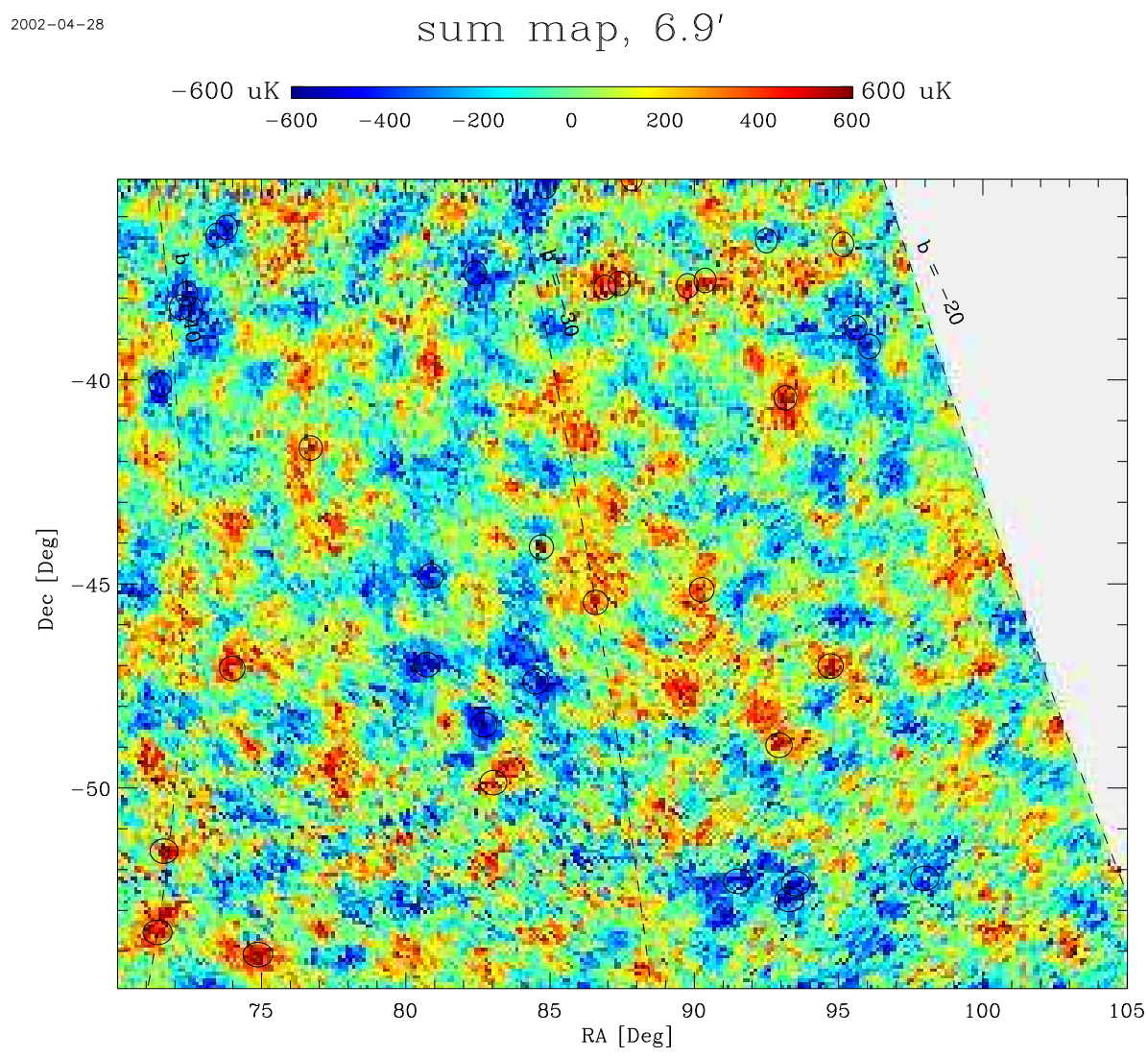

Fig. 1. Sum $(\mathrm{A}+\mathrm{B})$ map obtained from three independent measurement channels at $150 \mathrm{GHz}$ : $\mathrm{A}+\mathrm{B}=\mathrm{B} 150 \mathrm{~A}+(\mathrm{B} 150 \mathrm{~A} 1+\mathrm{B} 150 \mathrm{~A} 2) / 2$. The pixel size is 6.9 arc-min (Healpix nside $=512)$. The measurement units $(\mu K)$ refer to thermodynamic temperature fluctuations of a $2.73 \mathrm{~K}$ Blackbody. The circles locate the anisotropy spots with more than 3 pixels detected at a threshold level of $\pm 500 \mu K$ (see Tables 1(a) and 1(b)).

orientation of the elongations does not show any preferred direction. The behaviour of ellipticity vs threshold we find is consistent with the one due to the correlations detected in the same map by means of the power spectrum analysis. 29

\section{Complexity of CMB Maps}

The theory of algorithmic information provides tools to study the CMB maps and extract information on the general properties of the underlying dynamical systems without specification of cosmological models. Such a description is the Kolmogorov complexity of the anisotropy areas (spots) $\frac{17}{17}$ which is the amount of information required to determine uniquely a given object. The conditional complexity

$$
K(x \mid y)=\min [l(p)]
$$



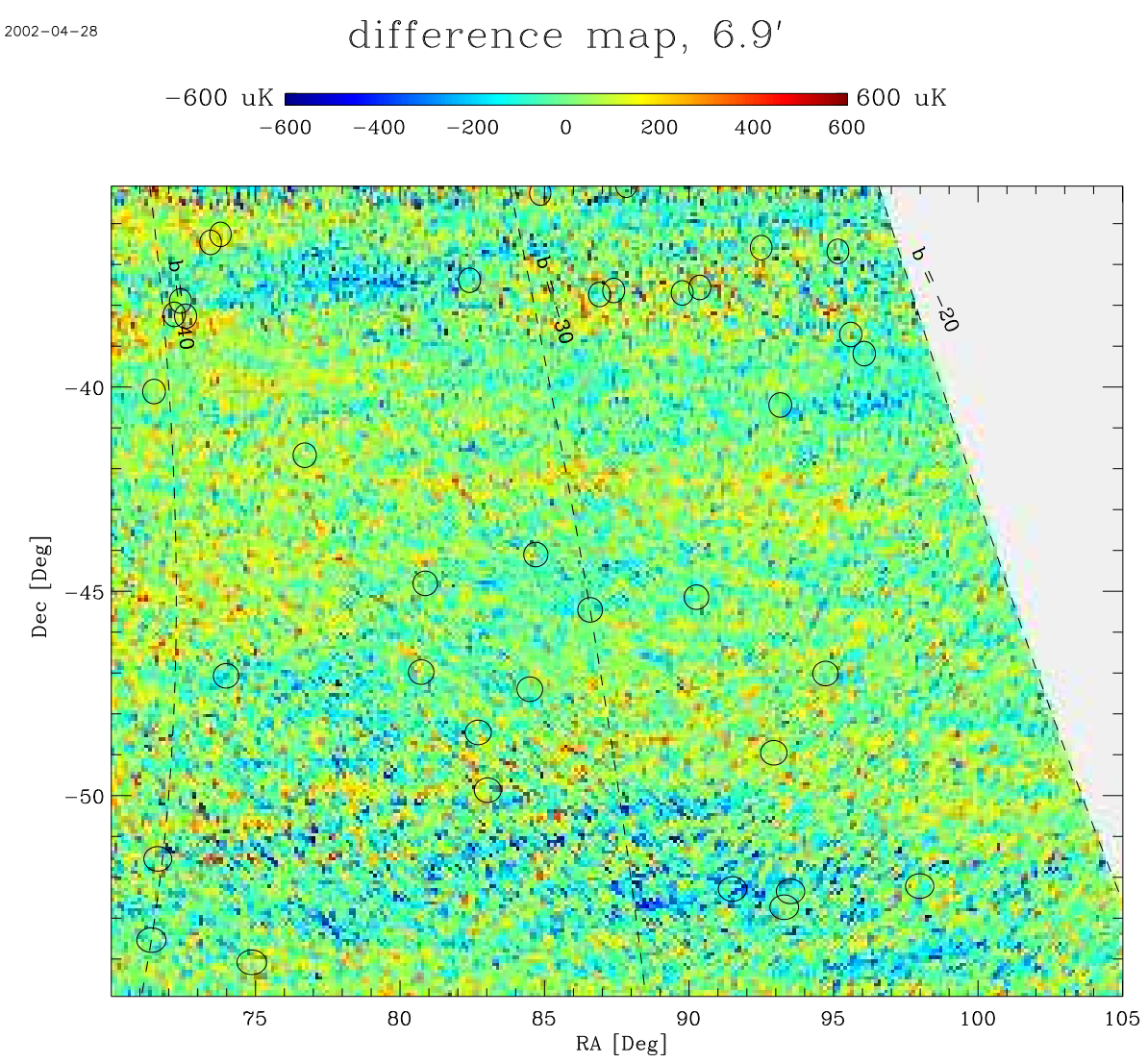

Fig. 2. Difference $(\mathrm{A}-\mathrm{B})$ map obtained from three independent measurement channels at $150 \mathrm{GHz}: \mathrm{A}-\mathrm{B}=\mathrm{B} 150 \mathrm{~A}-(\mathrm{B} 150 \mathrm{~A} 1+\mathrm{B} 150 \mathrm{~A} 2) / 2$. The pixel size is 6.9 arc-min (Healpix nside $=512)$. The measurement units $(\mu K)$ refer to thermodynamic temperature fluctuations of a $2.73 \mathrm{~K}$ blackbody. The circles locate the anisotropy spots with more than 3 pixels detected in the sum $(\mathrm{A}+\mathrm{B})$ map at a threshold level of $\pm 500 \mu K$ (see Tables $1(\mathrm{a})$ and 1(b)).

is related to the the amount of information on the object $x$ with respect the object 1022

$$
I(y: x)=K(x)-K(x \mid y),
$$

and is the minimal length $l(p)$ of the binary coded program required to describe the object $x$ when the one for $y$ is known. The complexity is related to KolmogorovSinai (KS) entropy $h$ via the relation

$$
K_{u}(t)-K_{u}\left(t_{0}\right)=\log _{2} 2^{h\left(f^{t}\right)\left(t-t_{0}\right)}=h\left(f^{t}\right)\left(t-t_{0}\right),
$$

quantifying the loss of information $\Delta I$ at the evolution of CMB pattern from the initial state $t_{0}$ up to $t$, i.e. from the last scattering epoch up to the observer. The ellipticity of anisotropy areas at each temperature threshold is the simplest description of the complexity of the areas. The ellipticity $\epsilon$ is defined via the divergence of 
the null geodesics in $(3+1)$-space

$$
\epsilon=\frac{L(t)}{L\left(t_{0}\right)}
$$

where

$$
L(t)=L\left(t_{0}\right) \frac{a(t)}{a\left(t_{0}\right)} \exp (h s),
$$

$a(t)$ is the scale factor of the Universe, $s$ is the affine parameter of the geodesics and KS-entropy is the sum of the positive Lyapunov exponents, $\frac{18}{18}$ for more properties of the geodesics (see Refs. 1, 19] and 25). The geodesic mixing is a statistical effect arising due to the exponential mixing, i.e. exponential decay of time correlation functions of the freely propagating photon beams at $k=-1$, is independent of the conditions on the last scattering surface, and is distinguished by the threshold independence and the randomness of the obliquities of the elongated areas. The ellipticity due to geodesics mixing has to vanish at precisely flat $k=0$ and positively curved, $k=+1$, spaces. $^{\text {a }}$

\section{Data}

BOOMERanG is a millimetric telescope with bolometric detectors on a balloon borne platform 1132 It was flown in 1998/99 and produced wide (4\% of the sky), high resolution $\left(\sim 10^{\prime}\right)$ maps of the microwave sky (90 to $\left.410 \mathrm{GHz}\right){ }^{12}$ Two observation modes were used to map this sky patch: sky scans at a speed of $1^{\circ} / \mathrm{s}$ and sky scans at $2^{\circ} / \mathrm{s}$. This allows us to perform powerful tests for systematics ${ }^{[29}$ In fact, at $2^{\circ} / \mathrm{s}$, the sky temperature distribution produces signals in the detection chain at frequencies which are doubled with respect to observations at $1 \%$, while instrument related effects, like $1 / \mathrm{f}$ noise, microphonic lines, and time-domain response remain at the same frequency. Comparing the maps obtained in the two observation modes is thus very effective in detecting instrumental artifacts. In the BOOMERanG maps the CMB structure is resolved with high signal to noise ratio, and hundreds of degree-scale hot and cold areas are evident. The rms temperature fluctuation of these areas is $\sim 80 \mu K$. The detected fluctuations are spectrally consistent with the derivative of a $2.735 \mathrm{~K}$ blackbody ${ }^{27}$ have shown that contamination from local foregrounds is negligible in the maps at 90,150 and $240 \mathrm{GHz}$, and that the $410 \mathrm{GHz}$ channel is a good monitor for dust emission. In our study we used two maps at $150 \mathrm{GHz}$. These maps have been obtained from the time ordered data using an iterative procedure,$\frac{31}{,}$ which properly takes into account the system noise and produces a maximum likelihood map. The largest structures (scales larger than $10^{\circ}$ ) are removed in this procedure, to avoid the dominating effects of instrument

a'We avoid the often used terms 'open' and 'closed' Universe, since the geometry does not define the topology, e.g. the flat, $k=0$ Universe can be not only $R^{3}$ but also $S^{1} \times R^{2}$, Tor $^{3}, R^{1} \times$ Tor $^{2}$, etc., and similarly the negatively curved $k=-1$ Universe can be both 'open' and 'closed'. 
drifts and $1 / f$ noise. The two input maps, $\mathrm{A}$ and $\mathrm{B}$, included 33111 pixels, each of $\sim 7$ arc-min in linear size, in a high Galactic latitude region covering about $1 \%$ of the sky, with coordinates $R A>70^{\circ},-55^{\circ}<\operatorname{dec}<-35^{\circ}$ and $b<-20^{\circ} 12$ The first map (A) has been obtained from the data of the B150A detector, while the second map (B) was obtained by averaging the maps from detectors B150A1 and B150A2. In this way we obtained two maps with similar noise per pixel. The (CMB) signal to noise ratio per pixel is of the order of 1 for our $7^{\prime}$ pixels. The sum $(\mathrm{A}+\mathrm{B})$ and difference $(\mathrm{A}-\mathrm{B})$ maps from all scans $\left(1^{\circ} / \mathrm{s}\right.$ and $\left.2^{\circ} / \mathrm{s}\right)$ are shown in Figs. 1 and 2 respectively. There are three AGN with significant flux in the maps (double circles in Figs. 1 and 2). This has been taken into account in the analysis.

\section{Analysis}

We studied the excursion sets in the BOOMERanG maps by means of a specially developed software ${ }^{20}$ This enabled, in an interactive way, to change the input parameters, like the threshold level and the minimum and maximum number of pixels forming an anisotropy area to be included in the analysis, and allowed the visualization of all the intermediate steps of the analysis.

\subsection{Algorithm}

To define the excursion sets (hereafter 'areas') in the maps, a matrix of temperature data of the pixels with equal and higher than the given temperature threshold (lower, for negative thresholds) has been formed and the contours of those areas have been studied. The original maps follow the Healpix pixelization scheme 16 However, the distance between pixel centers is not constant for this pixelization, so we had to regrid the maps. We also oversampled the maps in order to easy the algorithm of definition of the excursion sets. We have reprojected the data using both Cartesian and curvilinear coordinates. We found that for our purpose the two are equivalent, and we used the Cartesian coordinates for simplicity. This procedure enabled us to check the sensitivity of the ellipticity results with respect to the coordinate system and the cell size, for different total numbers of cells. In particular, an oversampled 1 arc-min cell (a $1692 \times 1296$ matrix) appears to lead to more accurate results than the original 7.5 arc-min cell matrix. The procedure of the definition of the centers of areas, their semi-axes and their obliquity and ellipticity was as follows:

(1) Center of the area. We define the coordinates of the center as $y_{c}=\left(y_{2}-y_{1}\right) / 2$, $x_{c}=\left(x_{2}-x_{1}\right) / 2$, where $y_{1}$ and $y_{2}$ are the highest and lowest $y$ coordinates of the pixels of the area (and analogously for $x$ coordinates).

(2) Semi-major axis $d_{\max }$. It is estimated as the segment connecting $\left(x_{c}, y_{c}\right)$ and the center of the farthest pixel of the area. The inclination angle of the segment is the obliquity, and is measured counter-clock-wise from the positive $x$ semi-axis (parallel to RA).

(3) Semi-minor axis $d_{\text {min }}$. On either sides of the major axis we find the pixels 
having the maximum distance between the pixel center and the major axis. We take the average of the two distances as the length of the semi-minor axis.

(4) The ellipticity is computed as $\epsilon=d_{\max } / d_{\min }$.

For each temperature threshold the mean ellipticity of the anisotropy areas was estimated for both the A $+\mathrm{B}$ and $\mathrm{A}-\mathrm{B}$ maps, along with the angular distribution of the obliquities. Our algorithm to estimate ellipticity is simpler and faster than the one used in Ref. 6] but, in the presence of noise, is biased, as we show below. The level of biasing, however, is reasonably small, and completely acceptable for our purpose.

\subsection{Simulations}

Since the use of ellipticity is relatively new in the CMB literature, we carried out numerical simulations in order to show the performance of our estimator and the expected behaviour for the map of the CMB. In order to validate our algorithm we produced simulated maps, with circular, symmetric Gaussians, well separated in the sky, with FWHM $\sim 30$ arc-min. We used the same pixelization used for the BOOMERanG data. The average ellipticity we measure is between 1.3 (lower thresholds) and 1.4 (higher thresholds). The deviation from the expected unit value is due to pixelization and to the algorithm used to define ellipticity. The result gets closer to 1 for larger FWHM. If we use Gaussians with two axis (minor 30 arc-

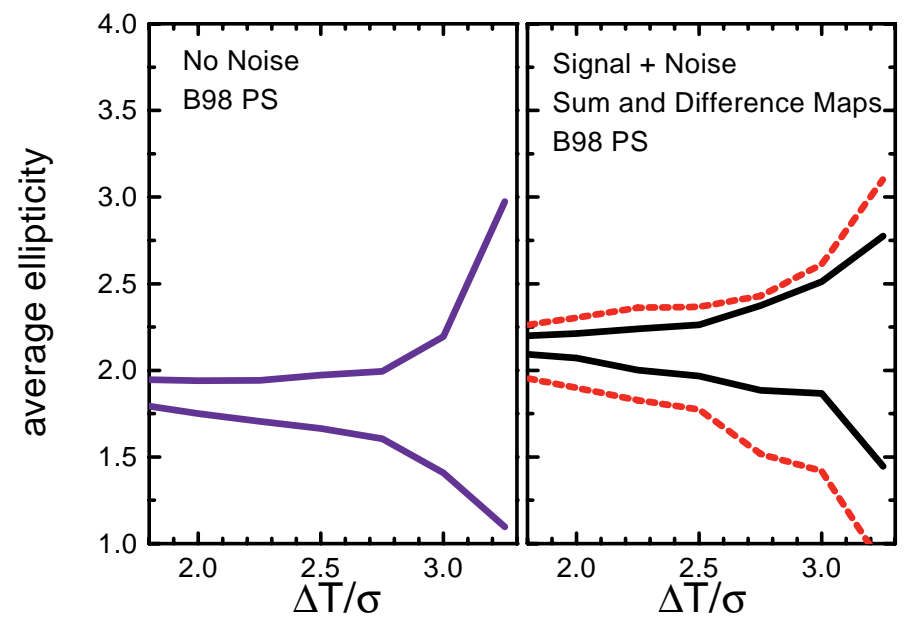

Fig. 3. Average ellipticity of intrinsic CMB anisotropy (left) obtained from simulations of CMB maps with the best fit power spectrum measured by BOOMERanG. The two lines define the $68 \%$ confidence interval for the map-averaged ellipticity measured from the simulations. In the right part of the figure we add realistic noise and filtering, at the same level present in the BOOMERanG $150 \mathrm{GHz}$ channels, and analyze the map-average ellipticity of simulations obtained summing (continuous lines) and differencing (dashed lines) maps from two independent measurement channels. The presence of noise increases the average ellipticity in the maps by $\sim 0.3$. In the difference maps, where only noise is present, the scatter of map-averaged ellipticities is higher. 


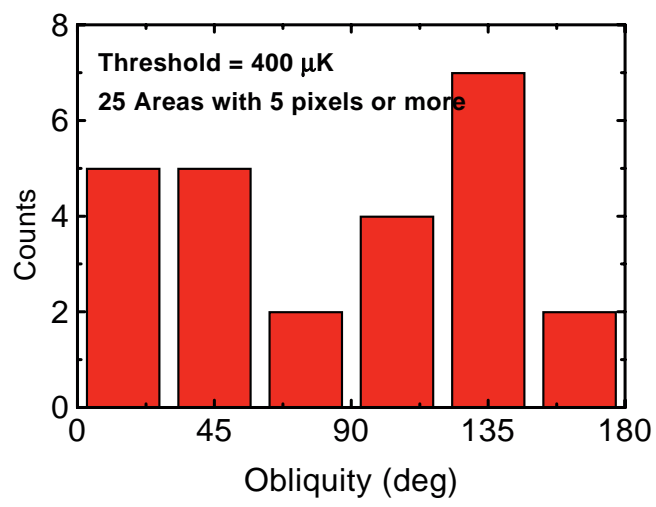

Fig. 4. Typical obliquity histogram for the A $+\mathrm{B}$ map. The threshold is $+400 \mu K$, and only areas with more than 4 pixels have been included.

min, major 45 arc-min), and we keep them well separated, we get an ellipticity of $\sim 1.8$, probably due to the bias we have seen before. Finally, we moved randomly the symmetric Gaussians, so that some of them merged and produced elliptical spots. In this case the mean ellipticity is a function of threshold, as expected. We conclude that our estimator of ellipticity is biased at a level of $\lesssim+0.4$ for spots smaller than $30^{\prime}$, and $\lesssim+0.1$ for spots larger than $1^{\circ}$. In Fig. 3 we illustrate the expected ellipticity behaviour of intrinsic CMB ellipticity in the context of the currently popular adiabatic inflationary model. Many realization of CMB maps of the sky region observed by BOOMERanG have been simulated, starting from the best fit angular power spectrum measured by BOOMERanG. For different temperature thresholds $T_{i}$, the anisotropy spots hotter than $T_{i}$ have been identified, and their ellipticities have been computed. In the left part of Fig. 3 the two lines define the $68 \%$ confidence intervals for the map-averaged ellipticity derived from these simulations. A map-averaged ellipticity $\sim 2$ is expected, basically independent of the temperature threshold. In the right part of Fig. 3 we simulate the presence of instrumental noise in the measurements of ellipticity, and show how to extract the CMB ellipticity signal from noisy sky maps. We have added realistic noise and filtering, at the same level present in the BOOMERanG $150 \mathrm{GHz}$ channels, and analyzed the map-average ellipticity of simulations obtained summing and differencing maps from two independent measurement channels. The presence of noise increases the level of the average ellipticity in the sum maps, at a level of about 0.3 . In the difference maps, where only noise is present, the scatter of map-averaged ellipticity is higher, as expected [8]

\subsection{Application to the BOOMERanG data}

Our software enabled to explicitly follow the evolution of anisotropy areas with respect the temperature threshold and the role of each area in the final results. 
As an example, Table 1 contains the data for the hot and cold anisotropy areas at thresholds $\pm 500 \mu K$. In Fig. 4 we plot a histogram of obliquity of the spots detected in the sum map $\mathrm{A}+\mathrm{B}$, at a temperature threshold of $+400 \mu K$. Only areas formed by 5 pixels or more have been considered, because the obliquity error due to pixelization is very large for areas with less pixels. The obliquities of these 25 areas are random. The $\chi^{2}$ for a uniform distribution is 6.13 with 5 DOF. The same typical behaviour has been found at different thresholds. Only when areas with relatively small number of pixels are included, there is a certain domination of alignment on 45 and 135 degrees, which disappears when such areas are abandoned, thus indicating the role of the rectangular shapes of the pixels themselves. This test is a strong indication that the origin for the detected ellipticity cannot be instrumental. Effects related to detectors time constants or to the scan strategy should be strongly anisotropic, as our scans are all within $\pm 12^{\circ}$ from the DEC $=$ constant lines. In Fig. 5 we plot the map-averaged ellipticity versus the temperature threshold for both the A + B map (signal plus noise) and the A - B map (noise only). In Fig. 6 we plot the same for a previous data release with higher noise $(S / N$ of the order of 0.7 versus 1.0) and lower accuracy pointing reconstruction $\left(4.5^{\prime}\right.$ rms versus $2.5^{\prime} \mathrm{rms}$ ). These data have been considered in order to investigate if the results are robust against variations of the noise level and of the pointing accuracy. The use of perturbed data is a standard and powerful technique in the framework of theory of dynamical systems. ${ }^{2]}$ We use it in order to check at once the effect of perturbations like inaccuracy in pointing, timestream filtering, detector noise, which are different for the two data releases we compare. Only the areas containing 3 to 200 pixels have

Table 1(a). Threshold: $-500 \mu K, \mathrm{~A}+\mathrm{B}$.

\begin{tabular}{ccccc}
\hline $\begin{array}{c}\text { Area } \\
\text { No }\end{array}$ & $\begin{array}{c}\text { Coordinates, degree } \\
\ell\end{array}$ & $b$ & $\begin{array}{c}\text { Number } \\
\text { of pixels }\end{array}$ & Ellipticity \\
\hline C1 & 243.9 & -40.4 & 5 & 4.72 \\
C2 & 241.5 & -39.7 & 3 & 1.42 \\
C3 & 241.1 & -39.5 & 4 & 1.96 \\
C4 & 241.6 & -39.4 & 3 & 2.43 \\
C5 & 239.4 & -38.5 & 3 & 1.61 \\
C6 & 239.2 & -38.2 & 4 & 4.33 \\
C7 & 253.1 & -34.2 & 12 & 1.73 \\
C8 & 250.5 & -33.9 & 6 & 2.20 \\
C9 & 255.0 & -33.0 & 12 & 2.21 \\
C10 & 253.9 & -31.7 & 8 & 1.69 \\
C11 & 242.0 & -31.6 & 6 & 2.49 \\
C12 & 240.1 & -29.2 & 4 & 3.17 \\
C13 & 260.2 & -27.9 & 6 & 2.24 \\
C14 & 260.9 & -26.9 & 4 & 2.39 \\
C15 & 260.5 & -26.7 & 7 & 2.02 \\
C16 & 261.0 & -24.0 & 3 & 1.28 \\
C17 & 243.4 & -23.6 & 4 & 2.18 \\
C18 & 246.4 & -21.9 & 3 & 2.48 \\
C19 & 247.0 & -21.7 & 4 & 1.95 \\
\hline
\end{tabular}


Table 1(b). Threshold: $+500 \mu K, \mathrm{~A}+\mathrm{B}$.

\begin{tabular}{ccccc}
\hline $\begin{array}{c}\text { Area } \\
\text { No }\end{array}$ & $\begin{array}{c}\text { Coordinates, degree } \\
\ell\end{array}$ & $b$ & $\begin{array}{c}\text { Number } \\
\text { of pixels }\end{array}$ & Ellipticity \\
\hline H1 & 258.9 & -40.1 & 5 & 2.10 \\
H2 & 261.5 & -40.0 & 3 & 1.60 \\
H3 & 253.0 & -38.8 & 4 & 1.86 \\
H4 & 261.9 & -37.9 & 5 & 2.52 \\
H5 & 246.3 & -36.6 & 4 & 2.25 \\
H6 & 256.7 & -32.9 & 3 & 2.84 \\
H7 & 240.7 & -32.7 & 3 & 2.39 \\
H8 & 250.1 & -31.1 & 5 & 1.68 \\
H9 & 251.9 & -30.0 & 3 & 1.45 \\
H10 & 243.3 & -28.2 & 5 & 1.89 \\
H11 & 243.3 & -27.8 & 4 & 2.39 \\
H12 & 252.1 & -27.4 & 3 & 1.57 \\
H13 & 240.6 & -26.8 & 3 & 1.53 \\
H14 & 256.7 & -26.4 & 4 & 1.71 \\
H15 & 243.9 & -26.0 & 6 & 2.27 \\
H16 & 243.9 & -25.5 & 3 & 2.41 \\
H17 & 254.9 & -24.8 & 6 & 1.62 \\
H18 & 247.6 & -24.2 & 7 & 1.61 \\
H19 & 244.2 & -21.6 & 3 & 2.16 \\
\hline
\end{tabular}

Note to Table 1(b): $\overline{\text { Area H7 includes a known AGN. Other two AGNs }}$ are present in the map we have analyzed, but they fill less than 3 pixels and were discarded.

been used, since areas with 1 and 2 pixels introduce biases related to the shape of the pixels. The mean elongation has been computed only for thresholds containing at least two areas. Including areas with more than 200 pixels is not informative, since only a few of such structures are present, and their topological properties are expected to be different from those of smaller areas. The lower boundary (in absolute value) of the temperature threshold interval of interest was determined as the level where most of the areas already have shapes for which an ellipticity can be assigned. The upper boundary marks the threshold where the number of areas present in the map is not less than 3 . In Table 2 more detailed information on the properties of the areas as a function of the temperature threshold is given. In addition to ellipticity we report the number of areas versus threshold and the average size of the areas. The existence of a threshold independent region for the ellipticity from the A $+\mathrm{B}$ map is seen in both Fig. 5 (referring to the final maps) and Fig. 6 (referring to the higher noise and less accurate pointing). This means that this feature is robust against perturbations of the pointing and noise level. The data with higher noise level enabled one to compare some properties of $\mathrm{A}+\mathrm{B}$ and $\mathrm{A}-\mathrm{B}$, to reveal the role of the noise. We proceeded as follows. We defined 'equivalent' sets of areas for the $\mathrm{A}+\mathrm{B}$ and $\mathrm{A}-\mathrm{B}$ maps, respectively, by selecting the two temperature threshold intervals with the same number of areas contributing to our measurement of ellipticity. For example, for positive thresholds, the interval $[450,625]$ in the $\mathrm{A}+\mathrm{B}$ map must be compared to the interval $[300,400]$ in the $\mathrm{A}-\mathrm{B}$ map, 
since both intervals include from 5 to over 50 areas. This scheme defines threshold intervals $[-300,-450] \mu K$ and $[300,400] \mu K$ for $\mathrm{A}-\mathrm{B}$ and $[-475,-625] \mu K$ and $[450,625] \mu K$ for $\mathrm{A}+\mathrm{B}$, as seen in Fig. 5 . We compute the variance of the ellipticity in those threshold intervals, as $\sigma^{2}=\sum(\epsilon-\bar{\epsilon})^{2} /(N-1)$, where $N$ is the number of thresholds in the interval. We find that the peak to peak scatter, for negative thresholds, is over $5 \sigma$ for $\mathrm{A}-\mathrm{B}$, while is $3 \sigma$ for $\mathrm{A}+\mathrm{B}$, with the overwhelming majority of points inside the $2 \sigma$ interval. Another feature not evident from Figure 5 is the scatter in ellipticities at a given threshold. In the first case $(A+B)$ the distribution of areas ellipticities for a given threshold is in general narrower than in the second case $(\mathrm{A}-\mathrm{B})$ especially at high thresholds. For example, $\delta \epsilon=\epsilon_{\max }-\epsilon_{\min }$ equals 1.3 for the threshold corresponding to 6 areas in $\mathrm{A}+\mathrm{B},(-600 \mu \mathrm{K})$, while it is 2.8 for the threshold corresponding to 5 areas in $\mathrm{A}-\mathrm{B}(-400 \mu K)$. In other words, at high thresholds the areas in $\mathrm{A}+\mathrm{B}$ possess more homogeneous ellipticities with respect to the areas in $\mathrm{A}-\mathrm{B}$. It is remarkable that in their other properties the $\mathrm{A}+\mathrm{B}$ and $\mathrm{A}-\mathrm{B}$ areas show no qualitative difference. Namely, they show very similar properties in: (A) the variation of the number of spots vs threshold, (B) the dependence of the mean area sizes, in pixel numbers per area, on the threshold (which is very similar within the "equivalent" intervals), (C) the ellipticity dependence on the area size. It thus appears that the two sets have identical properties except for the scatter of ellipticity over the thresholds. This behavior of ellipticity is robust against the variation of the parameters. For example, the change of the minimal number of the pixels per area from 3 to $5,7,10$ does not change the properties of elongation in the A + B data. We also selected only the spots larger than 100 pixels (corresponding to few degrees sized spots) and found again an ellipticity $2.3-2.5$ with the same stability with respect to threshold we found for smaller spots. For these spots the bias due to noise and algorithm is smaller than +0.3 . The grand-average ellipticities for the final maps are reported in Table 3.

Table 2(a). Pixel range: $3-200, \mathrm{~A}+\mathrm{B}$ thresholds $<0$.

\begin{tabular}{ccccccc}
\hline Threshold $(\mu K)$ & Pixels & Areas & Pix/Ar & $\epsilon_{\min }$ & $\epsilon_{\max }$ & $\epsilon_{\text {mean }}$ \\
\hline-300 & 1669 & 180 & 9.30 & 1.28 & 6.72 & 2.25 \\
-325 & 1236 & 152 & 8.10 & 1.25 & 4.38 & 2.16 \\
-350 & 947 & 125 & 7.60 & 1.25 & 4.83 & 2.18 \\
-375 & 727 & 105 & 6.90 & 1.28 & 4.37 & 2.26 \\
-400 & 538 & 81 & 6.60 & 1.28 & 4.82 & 2.27 \\
-425 & 364 & 59 & 6.20 & 1.28 & 4.82 & 2.19 \\
-450 & 262 & 44 & 6.00 & 1.28 & 4.54 & 2.05 \\
-475 & 170 & 31 & 5.50 & 1.28 & 4.72 & 2.24 \\
-500 & 101 & 19 & 5.30 & 1.28 & 4.72 & 2.24 \\
-525 & 73 & 15 & 4.90 & 1.42 & 4.72 & 2.54 \\
-550 & 39 & 10 & 3.90 & 1.28 & 3.40 & 2.46 \\
-575 & 22 & 6 & 3.70 & 1.42 & 3.40 & 2.14 \\
-600 & 9 & 3 & 3.00 & 1.56 & 2.48 & 2.08 \\
-625 & 3 & 1 & 3.00 & 2.19 & 2.19 & 2.19 \\
-650 & 3 & 1 & 3.00 & 2.19 & 2.19 & 2.19 \\
\hline
\end{tabular}




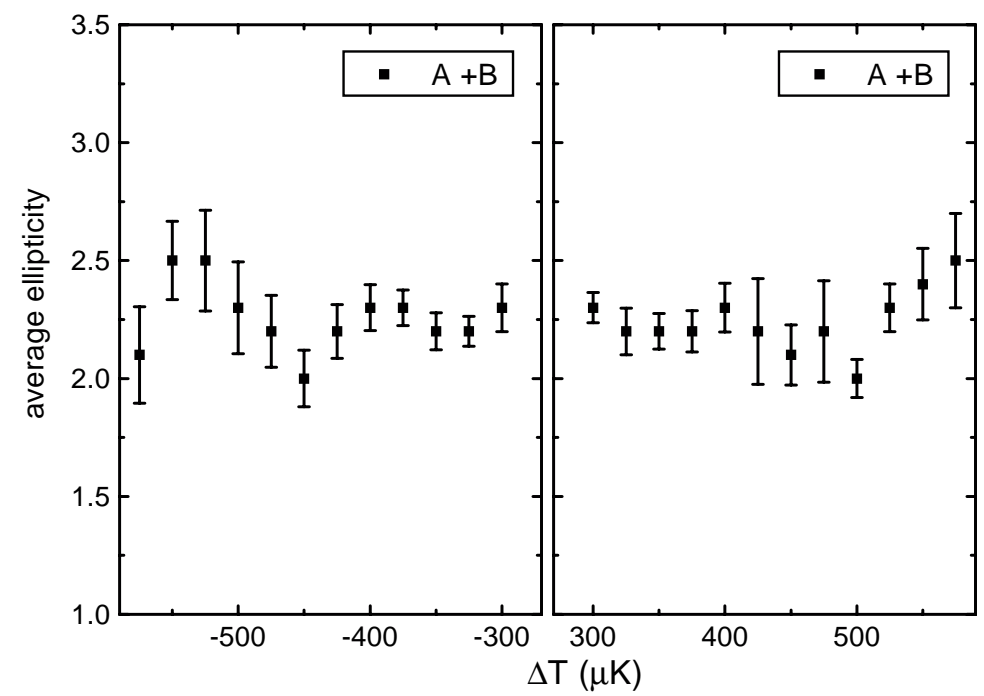

Fig. 5. Ellipticity vs temperature threshold (in $\mu K$ ) for sum $(\mathrm{A}+\mathrm{B})$ map at $150 \mathrm{GHz}$. Only areas containing more than 3 pixels and less than 200 in the final maps have been considered.

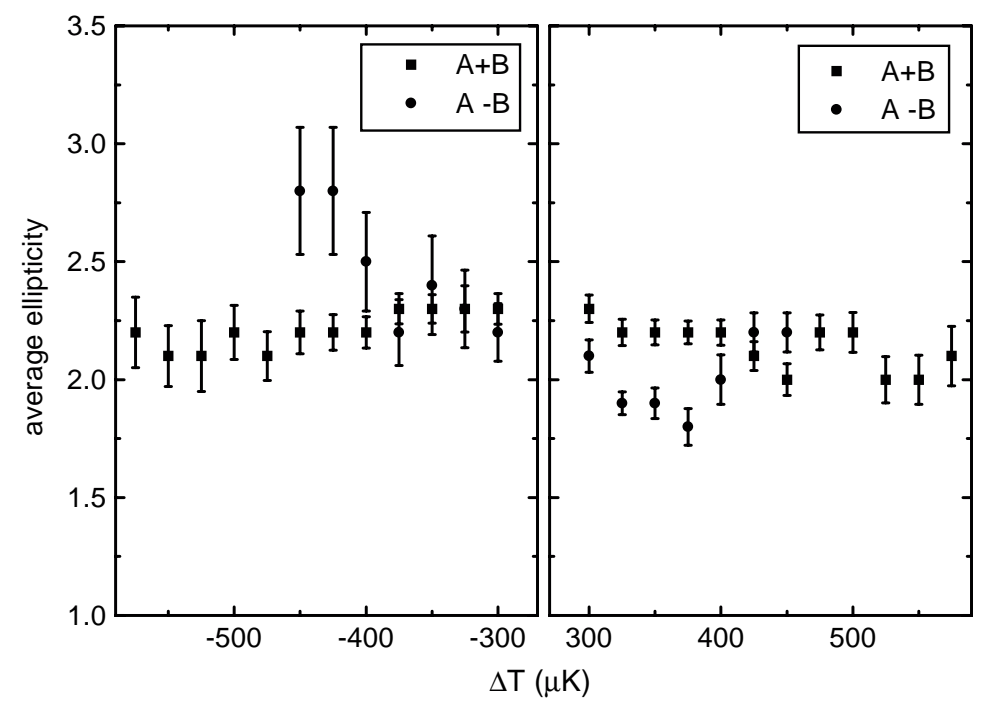

Fig. 6. Same as Fig. 5 for data with preliminary pointing reconstruction $\left(4.5^{\prime} \mathrm{rms}\right.$ pointing error instead of $\left.2.5^{\prime}\right)$ and lower signal to noise ratio (0.7 instead of 1$)$. Data for the difference maps are also plotted as circles.

\subsection{Systematics}

Possible systematic effect could be expected for very small areas due to the shape of the Healpix pixels. However, we find that this effect, for such small spots, is smeared by the intrinsic inaccuracy of the definition of the two semi-axes. We also checked 
Table 2(b). Pixel range pixel: 3-200, A + B thresholds $>0$.

\begin{tabular}{ccccccc}
\hline Threshold $(\mu K)$ & Pixels & Areas & Pix/Ar & $\epsilon_{\min }$ & $\epsilon_{\max }$ & $\epsilon_{\text {mean }}$ \\
\hline 300 & 1601 & 187 & 8.60 & 1.15 & 4.60 & 2.26 \\
325 & 1196 & 153 & 7.80 & 1.15 & 5.97 & 2.18 \\
350 & 899 & 134 & 6.70 & 1.29 & 4.77 & 2.20 \\
375 & 623 & 100 & 6.20 & 1.29 & 4.82 & 2.17 \\
400 & 426 & 76 & 5.60 & 1.29 & 4.93 & 2.30 \\
425 & 286 & 51 & 5.60 & 1.28 & 7.66 & 2.23 \\
450 & 178 & 37 & 4.80 & 1.28 & 4.37 & 2.09 \\
475 & 119 & 25 & 4.80 & 1.34 & 5.57 & 2.16 \\
500 & 79 & 19 & 4.20 & 1.45 & 2.84 & 1.99 \\
525 & 46 & 12 & 3.80 & 1.45 & 2.84 & 2.25 \\
550 & 25 & 7 & 3.60 & 1.60 & 3.27 & 2.44 \\
575 & 15 & 4 & 3.80 & 1.60 & 3.21 & 2.51 \\
600 & 6 & 2 & 3.00 & 1.42 & 1.60 & 1.51 \\
625 & 3 & 1 & 3.00 & 1.42 & 1.42 & 1.42 \\
650 & 3 & 1 & 3.00 & 1.42 & 1.42 & 1.42 \\
\hline
\end{tabular}

Table 3.

\begin{tabular}{lcc}
\hline Pixel interval per area & {$[3,200]$} & {$[3,200]$} \\
Threshold interval $(\mu K)$ & {$[-400,-600]$} & {$[375,575]$} \\
Number of areas & 268 & 331 \\
Mean ellipticity & $2.32 \pm 0.06$ & $2.22 \pm 0.05$ \\
\hline
\end{tabular}

Note to Table 3: The quoted errors are statistical only. A bias of $\lesssim 0.3$ due to the algorithm, and a similiar bias due to the noise have not been subtracted.

the effect of including data closer to the Galactic plane (down to $b<-13^{\circ}$ ). We found that the flat ellipticity vs threshold behaviour was strongly distorted by the presence of low galactic latitude data. We repeated the analysis above separately for maps obtained from $1^{\circ} / \mathrm{s}$ scans only and for maps obtained from $2^{\circ} / \mathrm{s}$ only, and obtained consistent results. The difference map $\left(1^{\circ} / \mathrm{s}\right.$ map $-2^{\circ} / \mathrm{s}$ map) features a larger scatter of the map-averaged ellipticity vs threshold diagram, as expected for noise only. This test strongly excludes an instrumental origin of the ellipticity of the areas.

\section{Conclusions}

We have produced a catalog of hot and cold spots in the BOOMERanG CMB maps. The distribution of number of spots and of their areas vs temperature threshold has been computed and can be compared to the predictions of cosmological models. We also found a threshold independent elongation of the spots in the meaningful interval of temperature thresholds. Selecting areas with three and more pixels, within temperature thresholds for $[375,575] \mu K$ (hot areas), we obtained a mean ellipticity $2.22 \pm 0.05$; in the range $[-400,-600] \mu K$ (cold areas) we obtained a mean ellipticity $2.32 \pm 0.06$. The quoted errors are statistical only.

This is the first measurement of the average ellipticity of the CMB at this 
angular resolution. The fact that its value is around 2 and not 1 or 3 should be regarded as a non-trivial experimental result. The bias deriving from pixelization and algorithm is of the order of +0.3 , while the bias due to the noise is also $\sim 0.3$ for areas smaller than $30^{\prime}$. The ellipticities quoted above have not been corrected for these effects. For larger spots $\left(\sim 2^{\circ}\right.$ size $)$ the bias is smaller but the ellipticity remains $\sim 2.5$. This means that for large areas the measured ellipticity is slightly higher than expected in the standard model.

The obliquities of anisotropies are random. The threshold independent and random obliquity behaviors in the sum map are stable against pointing reconstruction accuracy and noise level of the data, thus confirming that these are actual properties of the dataset. The same description estimated for the COBE maps yielded $\epsilon_{\mathrm{COBE}}=1.88 \pm 0.17{ }^{21}$ The analysis described here, however, was based on a much larger number of areas than in COBE, and an adaptive software was used.

A priori there is no reason for a random field to produce a constant ellipticity vs threshold. The detected behavior has to be produced by the correlations present in the sky signal. The presence of correlations in the map is not surprising, as evident from the power spectrum of the maps, 29 and indeed our experimental results are consistent with the results of simulations (compare Fig. 3 to Fig. 5).

There is significant debate about the nature of the cosmological model after the recent WMAP release. 34 171426138 Non-precisely zero curvature is among the discussed reasons of the low power at low multipoles of the CMB. The accuracy of the data used here gives also a hint of high ellipticity for the largest spots. If this is due to geodesics mixing, it would also point to a non-zero curvature. This analysis can be within reach of the data from forthcoming experiments.

\section{Acknowledgments}

The BOOMERanG experiment is supported in Italy by Agenzia Spaziale Italiana, Programma Nazionale Ricerche in Antartide, Universita' di Roma La Sapienza; by PPARC in the UK, by NASA, NSF OPP and NERSC in the U.S.A., and by CIAR and NSERC in Canada.

\section{References}

1. D. V. Anosov, Comm. Steklov Mathematical Inst., Vol. 90 (1967).

2. V. I. Arnold, Mathematical Methods of Classical Mechanics (Springer, 1989).

3. R. Aurich and F. Steiner, astro-ph/0302264 (2003).

4. S. L. Bridle et al., Science 299, 1532 (2003).

5. J. M. Bardeen et al., Astrophys. J. 304, 15 (1986).

6. R. B. Barreiro et al., Mon. Not. R. Astron. Soc. 322, 411 (2001).

7. C. L. Bennett et al., Astrophys. J. Suppl. 148, 1 (2003).

8. J. R. Bond and G. Efstathiou, Mon. Not. R. Astron. Soc. 226, 655 (1987).

9. L. Cayón and G. F. Smoot, Astrophys. J. 452, 487 (1995).

10. A. Chaitin, Algorithmic Information Theory (Cambridge University Press, 1987).

11. B. P. Crill et al., astro-ph//0206254, see also Ph.D. thesis, Caltech, 2000. 
12. P. de Bernardis et al., Nature 404, 955 (2000).

13. P. de Bernardis et al., Astrophys. J. 564, 559 (2002).

14. G. Efstathiou, 343, L95 (2003).

15. W. K. Hensinger et al., Nature 412, 52 (2001).

16. K. M. Górski, E. Hivon and B. D. Wandelt, astro-ph/9812350 (1998); http://www.eso.org/kgorski/healpix/

17. V. G. Gurzadyan, Europhys. Lett. 46, 114 (1999).

18. V. G. Gurzadyan and A. A. Kocharyan, Astron \& Astrophys. 260, 14 (1992); Europhys. Lett. 22, 231 (1993).

19. V. G. Gurzadyan and A. A. Kocharyan, Paradigms of the Large-Scale Universe (Gordon and Breach, 1994).

20. V. G. Gurzadyan and A. L. Kashin, Proc. IX Marcel Grossmann Meeting (World Scientific, Singapore, 2002), p. 2167.

21. V. G. Gurzadyan and S. Torres, Astron. \& Astrophys. 321, 19 (1997).

22. A. N. Kolmogorov, UMN 38, 97 (1983).

23. A. Lee et al., Astrophys. J. 561, L1 (2002).

24. E. M. Leitch et al., Astrophys. J. 568, 28 (2002).

25. C. M. Lockhart, B. Misra and I. Prigogine, Phys. Rev. D25, 921 (1982).

26. J.-P. Luminet et al., Nature 425, 593 (2003).

27. S. Masi, et al., Astrophys. J. 553, L93 (2001).

28. B. Mason et al., Astrophys. J. 591, 540 (2002); T. J. Pearson et al., Astrophys. J. 591, 556 (2002).

29. C. B. Netterfield et al., Astrophys. J. 571, 604 (2002).

30. D. Novikov, J. Schmalzing and V. F. Mukhanov, Astron. \&S Astrophys. 364, 17 (2000).

31. P. Natoli et al., Astron. \& Astrophys. 371, 346 (2001).

32. F. Piacentini et al., Astrophys. J. Suppl. 138, 315 (2002).

33. G. Polenta et al., Astrophys. J. 572, L27 (2002).

34. P. F. Scott et al., Mon. Not. R. Astron. Soc. 341, 1057 (2002).

35. J. C. Sommerer and E. Ott, Science 259, 335 (1993).

36. D. A. Steck, W. H. Osay and M. G. Raizen, Science 293, 274 (2001).

37. S. Torres, Astrophys. J. 423, L9 (1994).

38. J.-P. Uzan, U. Kirchner and G. F. R. Ellis, Mon. Not. R. Astron. Soc. 344, L65 (2003).

39. J. H. P. Wu et al., Phys. Rev. Lett. 87, 251303 (2001). 